\title{
Baryonic Acoustic Oscillations via the Renormalization Group
}

\author{
Sabino Matarrese* \\ Dipartimento di Fisica "G. Galilei," Università di Padova, \\ and INFN, Sezione di Padova, via Marzolo 8, Padova I-35131, Italy \\ Massimo Pietroni ${ }^{\dagger}$ \\ INFN, Sezione di Padova, via Marzolo 8, I-35131, Italy
}

(Dated: September 25, 2018)

\begin{abstract}
A semi-analytic approach to the computation of the non-linear power-spectrum of dark matter density fluctuations is proposed. The method is based on the Renormalization Group technique and can be applied to any underlying cosmological model. Our prediction on the baryonic acoustic oscillations in a $\Lambda \mathrm{CDM}$ model accurately fits the results of $\mathrm{N}$-body simulations down to zero redshift, where perturbation theory fails.
\end{abstract}

PACS numbers: 98.80.-k

Cosmological linear perturbation theory is a fundamental tool in the physics of the Cosmic Microwave Background (CMB), allowing a careful extraction of cosmological parameters from the data. In the case of LargeScale Structure, the accuracy of theoretical predictions is not yet at the same level than for CMB, due to the higher degree of non-linearity of the underlying density fluctuations. For instance, the study of Baryonic Acoustic Oscillations (BAO) - a fundamental probe for Dark Energy measurements - requires a precision of a few percent in the theoretical predictions for the matter powerspectrum (PS) in the wavenumber range $k \simeq 0.05-0.25$ $\mathrm{h} / \mathrm{Mpc}$ [1]. Including higher orders in PT [2] is known to give a poor performance in this range, leaving N-body simulations as the only viable approach to the problem.

Recently, however, perturbation theory (PT) has experienced a renewed interest, mainly motivated by two reasons. First, next generation galaxy surveys are going to measure the PS at large redshift, where the fluctuations are still in the linear regime and 1-loop PT is expected to work [1]. Second, Crocce and Scoccimarro [3] have shown that the perturbative expansion can be reorganized in a very convenient way, which allows the use of standard tools of field theory, like the Feynman diagrams. They managed to compute the two-point correlator between density or velocity field fluctuations at different times (the 'propagator') by resumming an infinite class of diagrams at all orders in PT. Other approaches can be found in Ref. [4].

In this Letter, we will proceed along the same path, by implementing Wilsonian Renormalization Group (RG) techniques to compute the PS. RG methods, widely used in statistical mechanics and quantum field theory [5], are particularly suited to physical situations in which there is a separation between the scale where one is supposed to control the 'fundamental' theory and the scale were measurements are actually made. Starting from the fundamental scale, the RG flow describes the gradual inclusion of fluctuations at scales closer and closer to the one relevant to measurements. The new fluctuations which are included at an intermediate step, feel an effective theory, which has been 'dressed' by the fluctuations already included. In the present case, the RG flow will start from small wavenumbers $k$, where linear theory works, to reach higher and higher $k$.

We will consider a self-gravitating system of Dark Matter (DM) particles which, in the "single-stream" approximation, is governed by the continuity, $\partial_{\tau} \delta+\nabla$. $[(1+\delta) \mathbf{v}]=0$, and Euler equations $\partial_{\tau} \mathbf{v}+\mathcal{H} \mathbf{v}+(\mathbf{v} \cdot \nabla) \mathbf{v}=$ $-\nabla \phi$. Here $\tau$ is the conformal time, $\delta$ the mass-density fluctuation, $\mathbf{v}$ the peculiar velocity, $\phi$ the peculiar gravitational potential which, on subhorizon scales, obeys the Poisson equation $\nabla^{2} \phi=\frac{3}{2} \mathcal{H}^{2} \delta$, with $\mathcal{H}=d \ln a / d \tau$, having assumed an Einstein-de Sitter background cosmology.

Following Ref. [3] we introduce the doublet $\varphi_{a}(\mathbf{k}, \eta)$ $(a=1,2)$ - defined in Fourier space - given by $\left(\varphi_{1}, \varphi_{2}\right) \equiv$ $e^{-\eta}(\delta,-i \mathbf{k} \cdot \mathbf{v} / \mathcal{H})$, where the time variable has been replaced by the logarithm of the scale factor, $\eta=\ln \left(a / a_{i n}\right)$, $a_{\text {in }}$ being the scale factor at a conveniently remote epoch, when all the relevant scales are well in the linear regime. Notice that, compared with the definition in Ref. [3], we have an overall factor $e^{-\eta}$, such that the linear growing mode corresponds to $\varphi_{a}=$ const. We can then rewrite the continuity and Euler equations in compact form, as

$$
\begin{aligned}
\left(\delta_{a b} \partial_{\eta}+\Omega_{a b}\right) \varphi_{b}(\mathbf{k}, \eta) & =e^{\eta} \gamma_{a b c}(\mathbf{k},-\mathbf{p},-\mathbf{q}) \\
& \times \varphi_{b}(\mathbf{p}, \eta) \varphi_{c}(\mathbf{q}, \eta),
\end{aligned}
$$

where $\boldsymbol{\Omega}=\left(\begin{array}{cc}1 & -1 \\ -3 / 2 & 3 / 2\end{array}\right)$ and repeated indices/momenta are summed/integrated over.

We have defined a vertex function, $\gamma_{a b c}(\mathbf{k}, \mathbf{p}, \mathbf{q})$ $(a, b, c,=1,2)$ whose only non-vanishing elements are $\gamma_{121}(\mathbf{k}, \mathbf{p}, \mathbf{q})=1 / 2 \delta_{D}(\mathbf{k}+\mathbf{p}+\mathbf{q}) \alpha(\mathbf{p}, \mathbf{q}), \gamma_{222}(\mathbf{k}, \mathbf{p}, \mathbf{q})=$ $\delta_{D}(\mathbf{k}+\mathbf{p}+\mathbf{q}) \beta(\mathbf{p}, \mathbf{q})$, and $\gamma_{121}(\mathbf{k}, \mathbf{p}, \mathbf{q})=\gamma_{112}(\mathbf{k}, \mathbf{q}, \mathbf{p})$, where $\alpha(\mathbf{p}, \mathbf{q})=[(\mathbf{p}+\mathbf{q}) \cdot \mathbf{p}] / p^{2}$ and $\beta(\mathbf{p}, \mathbf{q})=(\mathbf{p}+\mathbf{q})^{2}(\mathbf{p}$. q) $/\left(2 p^{2} q^{2}\right)$. 
Besides the vertex, the other relevant dynamical quantity is the linear retarded propagator, which gives the linear evolution in $\eta$ of $\varphi_{a}, \varphi_{a}^{0}(\mathbf{k}, \eta)=g_{a b}\left(\eta, \eta^{\prime}\right) \varphi_{b}^{0}\left(\mathbf{k}, \eta^{\prime}\right)$, with $\eta>\eta^{\prime}$ (the subscript " 0 " indicates solutions of the linear equations, $\left.e^{\eta} \gamma_{a b c}=0\right)$, which reads $g_{a b}\left(\eta, \eta^{\prime}\right)=$ $\left[\mathbf{B}+\mathbf{A} \exp \left(-5 / 2\left(\eta-\eta^{\prime}\right)\right)\right]_{a b} \theta\left(\eta-\eta^{\prime}\right)$, with $\theta$ the stepfunction, $\mathbf{B}=\frac{1}{5}\left(\begin{array}{ll}3 & 2 \\ 3 & 2\end{array}\right)$ and $\mathbf{A}=\frac{1}{5}\left(\begin{array}{rr}2 & -2 \\ -3 & 3\end{array}\right)$. The growing $\left(\varphi_{a} \propto\right.$ const.) and the decaying $\left(\varphi_{a} \propto\right.$ $\exp (-5 / 2 \eta))$ modes can be selected by considering initial fields $\varphi_{a}$ proportional to $u_{a}=(1,1)$ and $v_{a}=(1,-3 / 2)$, respectively.

To extend the validity of this approach to $\Lambda \mathrm{CDM}$, we reinterpret the variable $\eta$ as the logarithm of the linear growth factor of the growing mode, i.e. $\eta=\ln \left(D^{+} / D_{i n}^{+}\right)$. This approximation has been shown to accurately fit Nbody simulations for different cosmologies [1].

Our aim is to apply methods familiar in quantum field theory to construct generating functionals for quantities like the PS, bispectrum, etc. ... The starting point is to write down an action giving the equation of motion (1) at its extrema. One can realize that a new, auxiliary, doublet field $\chi_{a}$ has to be introduced to this aim, and that the action is given by

$$
\begin{aligned}
& S=\int d \eta\left[\chi_{a}(-\mathbf{k}, \eta)\left(\delta_{a b} \partial_{\eta}+\Omega_{a b}\right) \varphi_{b}(\mathbf{k}, \eta)\right. \\
& \left.-e^{\eta} \gamma_{a b c}(-\mathbf{k},-\mathbf{p},-\mathbf{q}) \chi_{a}(\mathbf{k}, \eta) \varphi_{b}(\mathbf{p}, \eta) \varphi_{c}(\mathbf{q}, \eta)\right]
\end{aligned}
$$

Varying the action $S$ w.r.t. $\chi_{a}$ gives precisely Eq. (1), while varying it w.r.t. $\varphi_{a}$ yields an equation solved by $\chi_{a}=0$.

The probability of a classical field configuration is a delta function centered on the solution of the equation of motion. By averaging over Gaussian initial conditions, one can show [6] that the generating functional of correlation functions involving the $\varphi$ and $\chi$ fields is the pathintegral

$$
\begin{aligned}
& Z\left[J_{a}, K_{b} ; P^{0}\right] \\
= & \int \mathcal{D} \varphi_{a} \mathcal{D} \chi_{b} \exp \left\{\int d \eta d \eta ^ { \prime } \left[-\frac{1}{2} \chi_{a} P_{a b}^{0} \delta(\eta) \delta\left(\eta^{\prime}\right) \chi_{b}\right.\right. \\
+ & \left.\left.i \chi_{a} g_{a b}^{-1} \varphi_{b}-i e^{\eta} \gamma_{a b c} \chi_{a} \varphi_{b} \varphi_{c}+i J_{a} \varphi_{a}+i K_{b} \chi_{b}\right]\right\},
\end{aligned}
$$

where $J_{a}$ and $K_{b}$ are external sources and the momentum dependence is everywhere implicit. Here $P_{a b}^{0}(k)=$ $P^{0}(k) u_{a} u_{b}$ and $P^{0}$ is the linear PS at the initial "time" $\eta=0$, having assumed pure growing-mode initial conditions. As usual, we define also the generator of connected Green's functions $W\left[J_{a}, K_{b}\right]=-i \ln Z\left[J_{a}, K_{b}\right]$ and, for 1-particle irreducible (1PI) ones, the effective action $\Gamma\left[\varphi_{a}, \chi_{b}\right]=W\left[J_{a}, K_{b}\right]-\int d \eta d^{3} \mathbf{k}\left(J_{a} \varphi_{a}+K_{b} \chi_{b}\right)$, in terms of the "classical" fields, $\varphi_{a}\left[J_{c}, K_{d}\right]=\delta W\left[J_{c}, K_{d}\right] / \delta J_{a}$ and $\chi_{b}\left[J_{c}, K_{d}\right]=\delta W\left[J_{c}, K_{d}\right] / \delta K_{b}$. In linear theory the path-

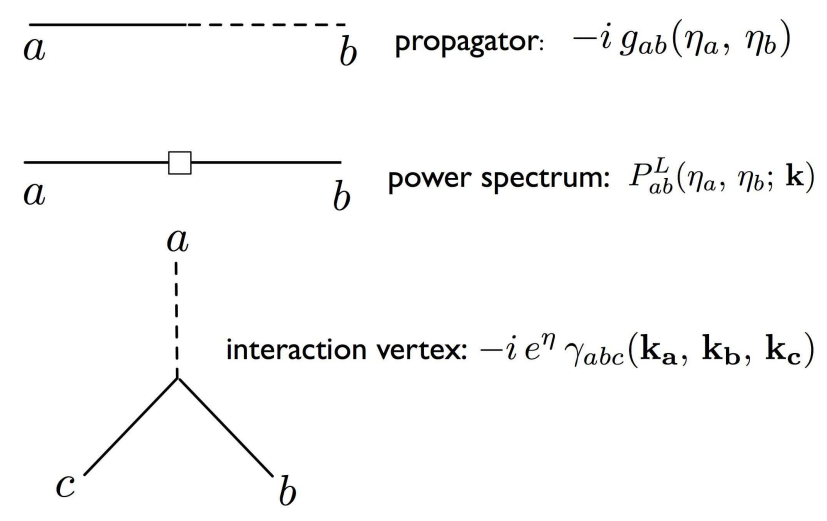

FIG. 1: Feynman rules

integral can be performed analytically,

$$
\begin{aligned}
Z_{0}\left[J_{a}, K_{b} ; P^{0}\right] & =\exp \left\{-\int d \eta d \eta^{\prime}\left[\frac{1}{2} J_{a}(\eta) P_{a b}^{L}\left(\eta, \eta^{\prime}\right) J_{b}\left(\eta^{\prime}\right)\right.\right. \\
& \left.\left.+i J_{a}(\eta) g_{a b}\left(\eta, \eta^{\prime}\right) K_{b}\left(\eta^{\prime}\right)\right]\right\}
\end{aligned}
$$

with $P_{a b}^{L}\left(\eta_{a}, \eta_{b} ; k\right)=g_{a c}\left(\eta_{a}, 0\right) g_{b d}\left(\eta_{b}, 0\right) P_{c d}^{0}(k)$ the linearly evolved PS.

Standard methods of PT can be applied, using the Feynman rules sketched in Fig. 1. There are three building blocks: the linear propagator $g_{a b}$, the linear PS $P_{a b}^{L}$ and the vertex $e^{\eta} \gamma_{a b c}$. The Feynman diagrams constructed using these rules are in one-to-one correspondence with those of Ref. [3], and reproduce all the known results of $\mathrm{PT}[2]$.

The full propagator, PS and vertex can be obtained from $W$ and $\Gamma$ as $\left(\delta^{2} W / \delta J_{a} \delta K_{b}\right)_{J, K=0} \equiv-\delta(\mathbf{k}+$ $\left.\mathbf{k}^{\prime}\right) G_{a b}, \quad\left(\delta^{2} W / \delta J_{a} \delta J_{b}\right)_{J, K=0} \equiv i \delta\left(\mathbf{k}+\mathbf{k}^{\prime}\right) P_{a b}$ and $\left(\delta^{3} \Gamma / \delta \chi_{a} \delta \varphi_{b} \delta \varphi_{c}\right)_{\varphi, \chi=0} \equiv-\Gamma_{a b c}\left(\mathbf{k}_{a}+\mathbf{k}_{b}+\mathbf{k}_{c} ; \eta\right)$.

Using the definitions of effective action and classical fields above, one can show that the full PS has the structure $P_{a b}=P_{a b}^{I}+P_{a b}^{I I}$, where $P_{a b}^{I}\left(k ; \eta, \eta^{\prime}\right)=$ $G_{a c}(k ; \eta, 0) G_{b d}\left(k ; \eta^{\prime}, 0\right) P_{c d}^{0}(k)$ and $P_{a b}^{I I}\left(k ; \eta, \eta^{\prime}\right)=$ $\int_{0}^{\eta} d \eta^{\prime \prime} \int_{0}^{\eta^{\prime}} d \eta^{\prime \prime \prime} G_{a c}\left(k ; \eta, \eta^{\prime \prime}\right) G_{b d}\left(k ; \eta^{\prime}, \eta^{\prime \prime \prime}\right) \Phi_{c d}\left(k ; \eta^{\prime \prime}, \eta^{\prime \prime \prime}\right)$, where $\Phi_{a b}$ is defined through $\left(\delta^{2} \Gamma / \delta \chi_{a} \chi_{b}\right)_{\varphi, \chi=0} \equiv$ $i P_{a b}^{0}(k) \delta(\eta) \delta\left(\eta^{\prime}\right)+i \Phi_{a b}$.

The starting point of our formulation of the RG is a modification of the primordial PS appearing in Eq. (3), as $P^{0}(k) \rightarrow P_{\lambda}^{0}(k)=P^{0}(k) \theta(\lambda-k)$. Inserting the truncated PS in Eq. (3) yields a generating functional $Z_{\lambda}\left[J_{a}, K_{b} ; P^{0}\right] \equiv Z\left[J_{a}, K_{b} ; P_{\lambda}^{0}\right]$, that describes a fictitious Universe, whose statistics of initial data is modified by suppressing all fluctuations with wavenumber larger than $\lambda$. On the other hand, the dynamical content, encoded in the linear propagator and in the structure of the interaction, is left unchanged.

In the $\lambda \rightarrow \infty$ limit, all the fluctuations are included, and we recover the physical situation. Increasing the 


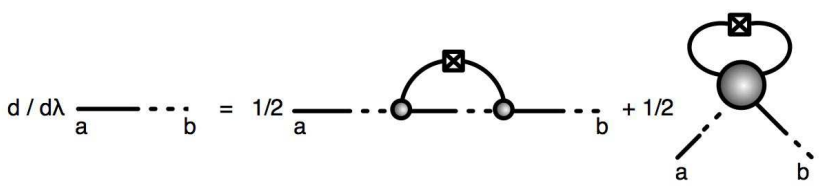

FIG. 2: RG equation for the propagator $G_{a b, \lambda}$

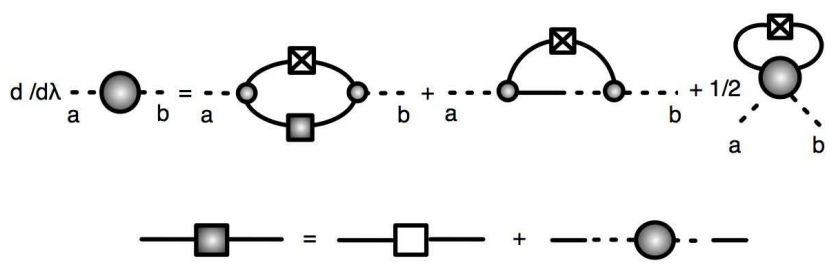

FIG. 3: RG equation for $\Phi_{a b, \lambda}$

cutoff from $\lambda=0$ to $\lambda \rightarrow \infty$, the linear and non-linear effect of fluctuations of higher and higher wavenumber is gradually taken into account. This process is described by a $\mathrm{RG}$ equation $[5,6]$ which can be obtained by taking the $\lambda$ derivative of $Z_{\lambda}$,

$\partial_{\lambda} Z_{\lambda}=\frac{1}{2} \int d \eta_{a} d \eta_{b} d^{3} \mathbf{q} \delta(\lambda-q) P_{a b}^{0}(q) \delta\left(\eta_{a}\right) \delta\left(\eta_{b}\right) \frac{\delta^{2} Z_{\lambda}}{\delta K_{b} \delta K_{a}}$.

Taking successive derivatives w.r.t. the sources of this master equation - or of the analogous ones for $W$ and $\Gamma$ - one can obtain the RG evolution of any physical quantity. The structure of the equation is such that the evolution of a correlation function of order $n$ involves all correlations up to order $n+2$. The RG equation for the propagator and for the function $\Phi_{a b, \lambda}$ are represented in Figs. 2 and 3, respectively, where the thick lines indicate full propagators, the dark box is the full PS $P_{a b, \lambda}$, dark blobs are full 1PI functions, and the crossed box is the RG kernel obtained by deriving w.r.t. $\lambda$ the $\theta$ function multiplying the PS in $P^{I}$, that is

$K_{a b, \lambda}\left(k, \eta, \eta^{\prime}\right)=G_{a c, \lambda}(k ; \eta, 0) G_{b d, \lambda}\left(k ; \eta^{\prime}, 0\right) P_{c d}^{0}(k) \delta(\lambda-k)$.

A recipe can be given to obtain the $\mathrm{RG}$ equation for any given quantity [6]:

i) write down the 1-loop expression for the quantity of interest, obtained using any needed vertex, (for instance, in Figs. 2, 3 we have not only the vertex $\chi \varphi \varphi$, but also $\chi \chi \varphi, \chi \varphi \varphi \varphi$ and $\chi \chi \varphi \varphi$, which vanish at tree-level);

ii) promote the linear propagator, the PS and the vertices appearing in that expression to full, $\lambda$-dependent ones;

iii) take the $\lambda$-derivative of the full expression, by considering only the explicit $\lambda$-dependence of the stepfunction contained in $P_{\lambda}^{I}$.

It should be emphasized that the RG equations obtained following these rules are exact, in the sense that they encode all the dynamical and statistical content of the path-integral (3) or, equivalently, of the continuity and Euler equations supplemented by the initial PS. Different approximation schemes can be attempted, including of course PT, which can be recovered by using quantities up to the $l$-th loop order in the RHS to get the $l+1$-th one by performing the $\lambda$-integration. However, our RG equations are most indicated for non-perturbative resummations.

As a first step, we note that the full 3 and 4-point functions appearing in the RG equations for $G_{a b, \lambda}$ and $\Phi_{a b, \lambda}$ are also $\lambda$-dependent quantities, which can be computed by RG equations, also derived from Eq. (5). These equations depends, in turn, on full, connected, and $\lambda$ dependent functions up to 5 (for the 3-point function) or 6 (for 4-point ones) external legs, which also evolve according to RG equations. Approximations to the full RG flow then amount to truncating the full hierarchy of coupled differential equations, and using some ansatz for the full n-point functions appearing in the surviving equations. We will approximate the full RG flow by keeping the running of the 2-point functions (propagator and PS) and keeping the tree-level expression for the trilinear vertex $\chi \varphi \varphi$. In this approximation, only the first diagrams on the RHS of Figs. 2 and 3 contribute to the running.

The 1-loop result for the propagator [2] can be recovered by using the tree-level expressions for the kernel $K_{a b, \lambda}$ and for the propagators on the RHS of Fig. 2.

Using running propagators on the RHS of Fig. 2, while keeping the kernel at tree-level, we get a RG equation which can be analytically integrated in the $k \gg q=\lambda$ limit,

$$
\begin{aligned}
\partial_{\lambda} G_{a b, \lambda}\left(k ; \eta_{a}, \eta_{b}\right)= & -G_{a b, \lambda}\left(k ; \eta_{a}, \eta_{b}\right) \frac{k^{2}}{3} \frac{\left(e^{\eta_{a}}-e^{\eta_{b}}\right)^{2}}{2} \\
& \times \int d^{3} \mathbf{q} \delta(\lambda-q) \frac{P^{0}(q)}{q^{2}}
\end{aligned}
$$

having used the property $u_{f} \gamma_{e f g}(-\mathbf{k}, \mathbf{q}, \mathbf{k}-\mathbf{q}) \simeq$ $\delta_{e g}(k / 2 q) \cos \mathbf{k} \cdot \mathbf{q}$, valid in this limit. Imposing the initial condition $G_{a b, \lambda=0}\left(k, \eta_{a}, \eta_{b}\right)=g_{a b}\left(\eta_{a}, \eta_{b}\right)$, and integrating up to $\lambda=\infty$, one gets

$$
G_{a b}\left(k, \eta_{a}, \eta_{b}\right)=g_{a b}\left(\eta_{a}, \eta_{b}\right) e^{-k^{2} \sigma_{v}^{2} \frac{\left(e^{\eta_{a}}-e^{\eta_{b}}\right)^{2}}{2}},
$$

where $\sigma_{v}^{2}$ is the velocity dispersion, defined as $\sigma_{v}^{2} \equiv$ $(1 / 3) \int d^{3} \mathbf{q} P^{0}(q) / q^{2}$. Two comments are in order. The $\mathrm{RG}$ improvement discussed here has a clear interpretation in terms of PT. Indeed, as shown in [3], the result in Eq. (8) can be obtained also by resumming an infinite class of diagrams. It is amazing how the same result, that in PT requires a careful control of the combinatorics, is here obtained by a simple, 1-loop, integration. The second comment has to do with the dramatic modification of the UV behavior of the resummed propagator w.r.t. the linear one, and on its impact on the RG flow. Indeed, when the propagator of Eq. (8) is employed in the kernel $K_{a b, \lambda}$, an intrinsic UV cut-off is provided to the 
RG flow: fluctuations with large momenta are exponentially damped, so that the RG evolution freezes out for $\lambda \gg e^{-\eta} / \sigma_{v}$. This is a genuinely non-perturbative effect, which is masked if one considers PT at any finite order. In other words, the full equations of motion are much better behaved in the UV than their perturbative approximations.

Improving further on the approximations leading to Eq. (8), we relax the $k \gg \lambda$ condition, keeping the full momentum dependence of the tree-level vertex, and using $\lambda$-dependent propagators also in the kernel (6). For the full propagator we use the ansatz $G_{a e, \lambda}\left(k, s_{1}, s_{2}\right)=$ $H_{a e, \lambda}(k) \exp \left[-k^{2} \sigma_{v}^{2} \frac{\left(e^{s_{1}}-e^{s_{2}}\right)^{2}}{2}\right]$, in which we have factored out the leading time behavior of Eq. (8). Inserting the expression above in the RG equations we obtain a closed system of four coupled differential equations, one for each component of $H_{a e, \lambda}$. In order to simplify it further, we consider the two combinations $G_{a 1, \lambda}+G_{a 2, \lambda}$, $(a=1,2)$, therefore, for each external momentum $k$, we solve a system of two coupled differential equations. Full details will be given in [6]. The exponential damping of Eq. (8) is exhibited also by our more refined scheme.

The RG evolution of the propagator governs that of the $P_{a b, \lambda}^{I}$ contribution to the full PS. On the other hand, the evolution of the other contribution, $P_{a b, \lambda}^{I I}$, can be computed by solving the equation in Fig. 3. We approximate the exact $\mathrm{RG}$ equations along the same lines we follow in the computation of the propagator. In particular, the $\eta$ integrations in the expression for $P_{a b}^{I I}$ require an ansatz for its 'time' dependence. In analogy with Eq. (8), we use, again on the RHS of the RG equation, $P_{a b, \lambda}^{I I}\left(q, \eta_{a}, \eta_{b}\right)=$ $\bar{P}_{a b, \lambda}^{I I}\left(q, s_{1}, s_{2}\right) \exp \left[-q^{2} \sigma_{v}^{2} \frac{\left(e^{\eta_{a}}-e^{s_{1}}\right)^{2}+\left(e^{\eta_{b}}-e^{s_{2}}\right)^{2}}{2}\right] . \quad$ We compute the PS at equal 'times', $\eta_{a}=\eta_{b}=\eta$, with the initial condition $P_{a b, \lambda=0}^{I I}(k)=0$. We consider a spatially flat $\Lambda$ CDM model with $\Omega_{\Lambda}^{0}=0.7, \Omega_{b}^{0}=0.046, h=0.72$, $n_{s}=1$. The primordial PS $P^{0}$ is taken from the output of linear theory at $z_{i n}=35$, as given by the CAMB Boltzmann code [7].

In Fig. 4 we plot our results for the PS (solid lines), in the momentum range relevant for the $\mathrm{BAO}$, at $z=0,1$, and 2. The short-dashed lines correspond to the linear theory and the long-dashed ones to 1-loop PT (which, at $z=0$ has been truncated for $k \gtrsim 0.17 \mathrm{~h} / \mathrm{Mpc}$, where $P^{I}$ takes negative values, signaling the breakdown of the perturbative expansion). The black squares are taken from the numerical simulations of Ref. [8]. To enhance the BAO feature, each PS has been divided by the linear one, in a model without baryons [9]. In the peak region, our $\mathrm{RG}$ results agree with those of $\mathrm{N}$-body simulations to a few percent accuracy down to redshift $z=0$, where linear and 1-loop perturbation theory badly fail. Thus, the dynamical behavior in this momentum range appears to be captured fairly well by the approximations implemented by our approach, namely the 'single stream approxima- tion', leading to Eq. (1), and the non-linear corrections of
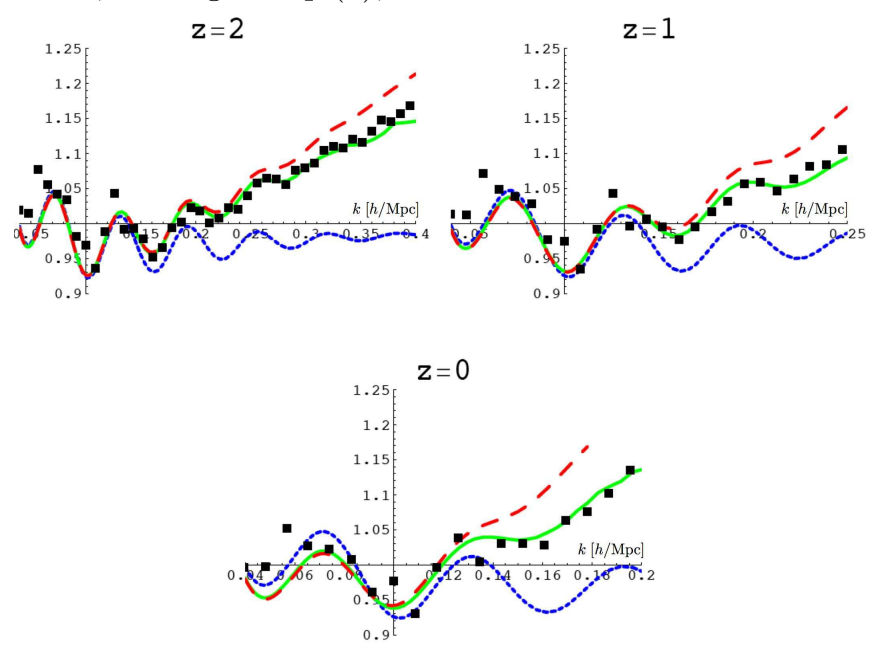

FIG. 4: The power-spectrum at $z=2,1,0$, as given by the RG (solid line), linear theory (short-dashed), 1-loop PT (longdashed), and the N-body simulations of [8] (squares).

the two-point functions only, i.e. the propagator and the PS. The RG performance can be systematically improved by increasing the level of truncation of the full tower of differential equations, the next step being the inclusion of the running of the trilinear vertex.

We thank M. White, for providing us with the N-body data of Ref. [8], N. Bartolo, P. McDonald and M. Viel, for discussions. M.P. thanks the Galileo Galilei Institute for Theoretical Physics for hospitality during the initial stages of this work.

* Electronic address: sabino.matarrese@pd.infn.it

$\dagger$ Electronic address: massimo.pietroni@pd.infn.it

[1] D. Jeong and E. Komatsu, Astrophys. J. 651, 619 (2006).

[2] F. Bernardeau, S. Colombi, E. Gaztanaga and R. Scoccimarro, Phys. Rept. 367, 1 (2002).

[3] M. Crocce and R. Scoccimarro, Phys. Rev. D 73, 063519 (2006); Phys. Rev. D 73, 063520 (2006).

[4] P. J. E. Peebles, Astrophys. J. 297, 350 (1985); P. McDonald, Phys. Rev. D 75, 043514 (2007); P. Valageas, Astron. Astrophys. 421, 23 (2004); P. Valageas, arXiv:astroph/0611849; T. Padmanabhan and S. Ray, Mon. Not. Roy. Astron. Soc. Lett. 372, L53 (2006); N. Afshordi, Phys. Rev. D 75, 021302 (2007).

[5] For a review on Wilsonian RG in QFT see T. R. Morris, Int. J. Mod. Phys. A 9, 2411 (1994).

[6] S. Matarrese and M. Pietroni, in preparation.

[7] A. Lewis, A. Challinor and A. Lasenby, Astrophys. J. 538, 473 (2000).

[8] E. Huff, A. E. Schulz, M. White, D. J. Schlegel and M. S. Warren, Astropart. Phys. 26, 351 (2007).

[9] D. J. Eisenstein and W. Hu, Astrophys. J. 496, 605 (1998). 